\title{
KP-CoT-23 (CCDC83) is a novel immunogenic cancer/testis antigen in colon cancer
}

\author{
MYUNG-HA SONG ${ }^{1 *}$, JIN-MOK HA $^{1 *}$, DONG-HOON SHIN ${ }^{2}$, \\ CHANG-HUN LEE ${ }^{2}$, LLOYD OLD $^{3 * *}$ and SANG-YULL LEE ${ }^{1}$ \\ Departments of ${ }^{1}$ Biochemistry and ${ }^{2}$ Pathology, School of Medicine, Pusan National University, \\ Yangsan-si, Gyeongsangnam-do 626-770, Republic of Korea; ${ }^{3}$ Ludwig Institute for Cancer Research, \\ New York Branch at Memorial Sloan-Kettering Cancer Center, New York, NY 10021, USA
}

Received May 10, 2012; Accepted July 16, 2012

DOI: 10.3892/ijo.2012.1601

\begin{abstract}
Cancer/testis (CT) antigens are considered target molecules for cancer immunotherapy. To identify novel CT antigens, immunoscreening of a testicular cDNA library was performed using serum obtained from a colon cancer patient who was immunized with a new dendritic cell vaccine. We isolated 64 positive cDNA clones comprised of 40 different genes, designated KP-CoT-1 through KP-CoT-40. Three of these putative antigens, including KP-CoT-23 (CCDC83), had testis-specific expression profiles in the Unigene database. RT-PCR analysis showed that the expression of 2 KP-Cot-23 variants was restricted to the testis in normal adult tissues. In addition, KP-CoT-23 variants were frequently expressed in a variety of tumors and cancer cell lines, including colon cancer. A serological western blot assay showed $\mathrm{IgG}$ antibodies to the KP-CoT-23 protein in 26 of 37 colon cancer patients and in 4 of 21 healthy patients. These data suggest that KP-CoT-23 is a novel CT antigen that may be useful for the diagnosis and immunotherapy of cancer.
\end{abstract}

\section{Introduction}

Cancer/testis (CT) antigens are immunogenic proteins with expression restricted to the testis and a wide range of human tumor types, eliciting both humoral and cellular immune responses in cancer patients (1). They are considered ideal targets for vaccine-based immunotherapy, and more than

Correspondence to: Professor Sang-Yull Lee, Department of Biochemistry, School of Medicine, Pusan National University, Beomeo-ri, Mulgeum-eup, Yangsan-si, Gyeongsangnam-do 626-770, Republic of Korea

E-mail: sangyull@pusan.ac.kr

*Contributed equally

${ }^{* *}$ Deceased

Key words: SEREX, cancer/testis antigen, colon cancer, KP-CoT-23, CCDC83
$100 \mathrm{CT}$ antigens, including MAGE, NY-ESO-1, GAGE, BAGE, LAGE, and SSX2, have been identified (2). CT antigens are divided into those that are encoded on the $\mathrm{X}$ chromosome (CT-X antigens) and those that are not (non-X CT antigens) (3). Many CT antigens exhibit heterogeneous expression patterns within the same tumor tissue $(2,4)$. Therefore, multiple CT antigens are needed to develop polyvalent cancer vaccines that overcome the limited frequency and heterogeneity of CT antigen expression. CT antigens have been identified through various techniques, including $\mathrm{T}$ cell epitope cloning, $\mathrm{MHC}$ peptide elution, differential gene expression analysis, and serologic expression cloning (SEREX) (5-8). Using DNA microarray analysis, MAA-1A was identified (9). Recently, massively parallel signature sequencing (MPSS) was utilized to compare the mRNA expression profiles of testis, melanoma cell lines, and other somatic tissues (10). This resulted in the identification of CT45. In addition to these experimental approaches, in silico analyses have also identified CT antigens, including BRDT (11), CT46 (12), PAGE (13), and XAGE1 (14).

Among all these methods, SEREX seems to be effective for the identification of CT antigens. SEREX screening of various cancer types was broadened to include screening of cDNA libraries derived from allogeneic tumors, tumor cell lines, and testis $(15,16)$. This investigation and similar studies by other researchers have led to the identification of more than 2,000 SEREX-defined antigens over several years (http://ludwigsun5.unil.ch/CancerImmunomeDB/). CT antigens identified by SEREX include MAGE-A (16), NY-ESO-1 (17), SSX2 (18), SCP1 (19), NY-SAR-35 (15), SLCO6A1 (20), CAGE-1 (21), and BCP-20 (22). In the present study, we performed SEREX analysis to screen a testicular cDNA library with the aim of isolating novel $\mathrm{CT}$ antigens. In addition to a previously defined CAGE CT antigen (23), a novel CT antigen, KP-CoT-23 (CCDC83), was identified.

\section{Materials and methods}

Human tissues, sera, and cell lines. Human tumor tissues and sera were obtained from the Department of Pathology, Pusan National University Hospital after diagnosis and staging. Tissues were frozen in liquid nitrogen and stored at $-80^{\circ} \mathrm{C}$ until use. The human colon cancer cell lines SNU-C1, 
SNU-C2A, SNU-C4, and SNU-C5; the human ovarian cancer cell lines SNU-8 and SNU-840; the human lung cancer cell lines SK-LC-5 and SK-LC-14; and the human breast cancer cell line MCF7 were obtained from the Korean Type Culture Collection (KTCC) and the American Type Culture Collection (ATCC). All these cell lines were maintained in RPMI-1640 medium (Gibco-BRL Life Technologies Inc., Grand Island, NY, USA) supplemented with $10 \%$ fetal bovine serum, $2 \mathrm{mM}$ l-glutamine, $100 \mathrm{U} / \mathrm{ml}$ penicillin, and $100 \mu \mathrm{g} / \mathrm{ml}$ streptomycin. The study was conducted under an approved protocol from Ethical Committeee in this institution.

Total RNA extraction from tissues and cell lines. Total RNA was isolated from human tissue samples and human tumor cell lines using the standard TRIzol reagent (Life Technologies, Gaithersburg, MD, USA) and RNA isolation kit (RNeasy Maxi Kit, Qiagen, Hilden, Germany) following the manufacturer's instructions. The amount of RNA isolated was measured at $260 \mathrm{~nm}$ by a spectrophotometer (Ultrospec 2000, Pharmacia Biotech, Cambridge, UK). Normal tissue total RNA was purchased from Clontech Laboratories, Inc. (Palo Alto, CA, USA) and Ambion, Inc. (Austin, Texas, USA). Total RNA from several cancer cell lines other than the ones used in this experiment was obtained from the Ludwig Institute for Cancer Research (LICR), New York Branch at the Memorial SloanKettering Cancer Center.

Preparation of cDNA library and sera. Poly(A)+ RNA from normal testis was purchased from Clontech Laboratories Inc. mRNA $(5 \mu \mathrm{g})$ was used to construct a cDNA library in the ZAP Express vector (Stratagene, La Jolla, CA, USA), following the manufacturer's instructions. The library contained approximately 1 million recombinants and was used for immunoscreening without prior amplification. Serum was prepared from a colon cancer patient and was diluted 1:200 for SEREX analysis. The colon cancer patient was immunized frequently (5 times) with a new dendritic cell vaccine (DC-Vac) into which autologous tumor lysate was loaded by electroporation and pulse. Serum from a human colon cancer patient was kindly provided by Dr Chi-Dug Kang (Pusan National University, Pusan City, Korea). The patient is a 56-year-old male with stage IV colon cancer. All sera from colon cancer patients and healthy individuals used in this study were diluted 1:200. To remove serum antibodies that react with Escherichia coli/bacteriophage-related antigens, sera were absorbed with $E$. coli/bacteriophage lysates as described by Lee et al (15).

Immunoscreening. Immunoscreening of the cDNA library was performed as previously described $(15,22)$. Briefly, E. coli XL1 blue MRF cells were transfected with the recombinant phages, and then plated at a density of approximately 5,000 pfu/150-mm plate (NZCYM-IPTG agar). The plates were incubated at $37^{\circ} \mathrm{C}$ for $8 \mathrm{~h}$, and transferred to nitrocellulose filters (Protran BA 85, $0.45 \mu \mathrm{m}$; Schleicher \& Schuell, Keene, NH, USA). The filters were then incubated with a 1:200 dilution of the patient's sera, which had been preabsorbed with E. coli-phage lysate. The serum-reactive clones were detected with an AP-conjugated secondary antibody and visualized by incubation with 5-bromo-4-chloro-3-indolyl-phosphate/nitro- blue tetrazolium (BCIP/NBT). After screening, the isolated positive clones were removed from the plate and preserved in suspension medium (SM) buffer with $25 \mu \mathrm{l}$ of chloroform. Positive phages were mixed with a helper phage to co-infect XL-1 Blue MRF, and they were rescued into pBluescript phagemid forms by in vivo excision. The excised phagemids were transformed into the host bacteria (XLOLR) to amplify plasmid DNA for extraction and stock. The size of the inserted cDNA was determined by restriction enzyme digestion with EcoRI and XhoI. The cDNA was sequenced commercially (Macrogen, Seoul, Korea).

$R T$-PCR. The cDNAs were prepared for use as templates for RT-PCR using the Superscript first strand synthesis kit (Invitrogen Life Technologies, Carlsbad, CA, USA) with $1 \mu \mathrm{g}$ of total RNA. The specific primers used for KP-CoT-23 were GCGATGAAGGAAAAATGGAA (forward) and GAGC CAGTCATTCTCCCAGATIIIIIGATAACTGC (reverse). For blocking extension of non-specifically primed templates and generating consistently high PCR specificity, we used a dual priming oligonucleotide (DPO) which contains two separate priming regions joined by a polydeoxyinosine linker (Segene Ins. Seoul, Korea). PCR primers used to verify the CCDC83 variants were GGA TGTTGAAGAAGCGATGAAGGA (forward) and CCAGGG GGCCCAAGTTTACA (reverse). The cDNA template concentrations were normalized to the amplification of GAPDH. For PCR, a $20 \mu \mathrm{l}$ reaction mixture containing $2 \mu \mathrm{l}$ of cDNA, $0.2 \mathrm{mM}$ dNTP, $1.5 \mathrm{mM} \mathrm{MgCl}_{2}$, $0.25 \mu \mathrm{M}$ each of the gene specific forward and reverse primers, and 3 units of Taq DNA polymerase (Solgent, Daejun, Korea) was preheated to $94^{\circ} \mathrm{C}$ for $5 \mathrm{~min}$, followed by 35 cycles at $94^{\circ} \mathrm{C}$ for $30 \mathrm{sec}, 60^{\circ} \mathrm{C}$ for $30 \mathrm{sec}$, and $72^{\circ} \mathrm{C}$ for $1 \mathrm{~min}$ followed by a final elongation step at $72^{\circ} \mathrm{C}$ for $5 \mathrm{~min}$. Amplified PCR products were analyzed on $1.5 \%$ agarose gels stained with ethidium bromide.

Generation of recombinant KP-CoT-23 fusion proteins. To generate His-tagged KP-CoT-23 proteins, we selected an open reading frame (ORF) cDNA from codons 514 to 1,748 within the CCDC83 ORF(NM_173556), including the target sequence for KP-CoT-23 variants. The PCR products contained the NdeI and XhoI restriction enzyme sites. The primers for the partial protein were GGAATTCCATATGGAAAACTCAGGG (forward) and CCGCTCGAGGAGAAAAGACTTCA (reverse). The PCR product was subcloned into the pET21a expression plasmid. E. coli BL21 cells containing the KP-CoT-23 plasmid were grown in LB liquid medium, and IPTG was added to a final concentration of $0.5 \mathrm{mM}$. Affinity chromatography using Ni-NTA resin (Qiagen) was performed to purify KP-CoT-23 recombinant protein. The purity of the recombinant protein was determined by SDS-PAGE and western blotting using anti-His antibody (Invitrogen Life Technologies) (Fig. 4C).

Western blot analysis. A $100 \mathrm{ng}$ of purified 6X His-KP-CoT-23 protein was separated on a $10 \%$ SDS-PAGE and transferred to a nitrocellulose membrane (Hybond-ECL; GE Healthcare, Little Chalfont, UK). After blocking with TBST (TBS and $0.1 \%$ Tween-20) containing 5\% skim milk for $1 \mathrm{~h}$ at room temperature, the membrane was incubated in sera (1:200 
Table I. Colon cancer antigens by SEREX.

\begin{tabular}{|c|c|c|c|}
\hline $\begin{array}{l}\text { No. of } \\
\text { Antigens }\end{array}$ & $\begin{array}{l}\text { Gene names/ } \\
\text { UniGene } \text { cluster }^{\mathrm{a}}\end{array}$ & $\begin{array}{l}\text { No. of } \\
\text { redundancies }\end{array}$ & $\begin{array}{c}\text { Previously } \\
\text { identified } \\
\text { by } \\
\text { SEREX }^{\mathrm{b}}\end{array}$ \\
\hline KP-CoT-1 & C6orf204/Hs.656359 & 1 & $\mathrm{Y}$ \\
\hline KP-CoT-2 & GKAP1/Hs.522255 & 15 & $\mathrm{~N}$ \\
\hline KP-CoT-3 & MRPS33/Hs.416207 & 1 & $\mathrm{Y}$ \\
\hline KP-CoT-4 & ANKRD50/Hs.480694 & 1 & $\mathrm{~N}$ \\
\hline KP-CoT-5 & CCDC19/Hs.647705 & 1 & $\mathrm{~N}$ \\
\hline KP-CoT-6 & BNC1/Hs.459153 & 1 & $\mathrm{~N}$ \\
\hline KP-CoT-7 & NACA/Hs.505735 & 1 & $\mathrm{Y}$ \\
\hline KP-CoT-8 & C16orf48/Hs.729159 & 1 & $\mathrm{Y}$ \\
\hline KP-CoT-9 & ZC3H15/Hs.731458 & 1 & $\mathrm{~N}$ \\
\hline KP-CoT-10 & COPB2/Hs.731508 & 2 & $\mathrm{~N}$ \\
\hline KP-CoT-11 & SPACA7/Hs.97592 & 2 & $\mathrm{~N}$ \\
\hline KP-CoT-12 & CEP290/Hs.150444 & 1 & $\mathrm{Y}$ \\
\hline KP-CoT-13 & RGPD5/Hs.469630 & 1 & $\mathrm{Y}$ \\
\hline KP-CoT-14 & IFT81/Hs.528382 & 1 & $\mathrm{~N}$ \\
\hline KP-CoT-15 & CIR1/Hs.632531 & 1 & $\mathrm{~N}$ \\
\hline KP-CoT-16 & TTC29/Hs.378893 & 1 & $\mathrm{~N}$ \\
\hline KP-CoT-17 & PLGLB1/Hs.652174 & 1 & $\mathrm{Y}$ \\
\hline KP-CoT-18 & BRAP/Hs.530940 & 6 & $\mathrm{Y}$ \\
\hline KP-CoT-19 & NAP1L3/Hs.21365 & 1 & $\mathrm{Y}$ \\
\hline KP-CoT-20 & PALLD/Hs.151220 & 1 & $\mathrm{Y}$ \\
\hline KP-CoT-21 & ETFB/Hs.348531 & 1 & $\mathrm{~N}$ \\
\hline KP-CoT-22 & LOC220115/Hs.528448 & 1 & $\mathrm{Y}$ \\
\hline KP-CoT-23 & CCDC83/Hs.567774 & 1 & $\mathrm{~N}$ \\
\hline KP-CoT-24 & NUPL1/Hs.732281 & 3 & $\mathrm{Y}$ \\
\hline KP-CoT-25 & TTC25/Hs.201134 & 1 & $\mathrm{~N}$ \\
\hline KP-CoT-26 & LRRC6/Hs.591865 & 2 & $\mathrm{~N}$ \\
\hline KP-CoT-27 & CAGE/Hs.434416 & 1 & $\mathrm{~N}$ \\
\hline KP-CoT-28 & KIAA0586/Hs.232532 & 1 & $\mathrm{Y}$ \\
\hline KP-CoT-29 & SUDS3/Hs.416630 & 1 & $\mathrm{Y}$ \\
\hline KP-CoT-30 & CMTM2/Hs.195685 & 1 & $\mathrm{~N}$ \\
\hline KP-CoT-31 & PIBF1/Hs.441926 & 1 & $\mathrm{~N}$ \\
\hline KP-CoT-32 & HSF2/Hs.158195 & 1 & $\mathrm{Y}$ \\
\hline KP-CoT-33 & C1orf55/Hs.520192 & 1 & $\mathrm{~N}$ \\
\hline KP-CoT-34 & CKM/Hs.334347 & 1 & $\mathrm{~N}$ \\
\hline KP-CoT-35 & EDDM3A/Hs.304757 & 1 & $\mathrm{~N}$ \\
\hline KP-CoT-36 & ATP6V1G1/Hs.388654 & 1 & $\mathrm{~N}$ \\
\hline KP-CoT-37 & DYNLRB2/Hs.98849 & 1 & $\mathrm{Y}$ \\
\hline KP-CoT-38 & RPL23A/Hs.419463 & 1 & $\mathrm{Y}$ \\
\hline KP-CoT-39 & H3F3B/Hs.180877 & 1 & $\mathrm{~N}$ \\
\hline KP-CoT-40 & POLR2J/Hs.654952 & 1 & $\mathrm{~N}$ \\
\hline
\end{tabular}

${ }^{a}$ UniGene cluster of isolated antigens (http://www.ncbi.nim.nih.gov/). ${ }^{\mathrm{b}}$ Sequences were compared with those contained in the SEREX database of the Ludwig Institute for Cancer Research (http://ludwigsun5. unil.ch/CancerImmunomeDB/). $\mathrm{Y}$ and $\mathrm{N}$ indicate whether the antigen matched or did not match an antigen in the SEREX database, respectively.

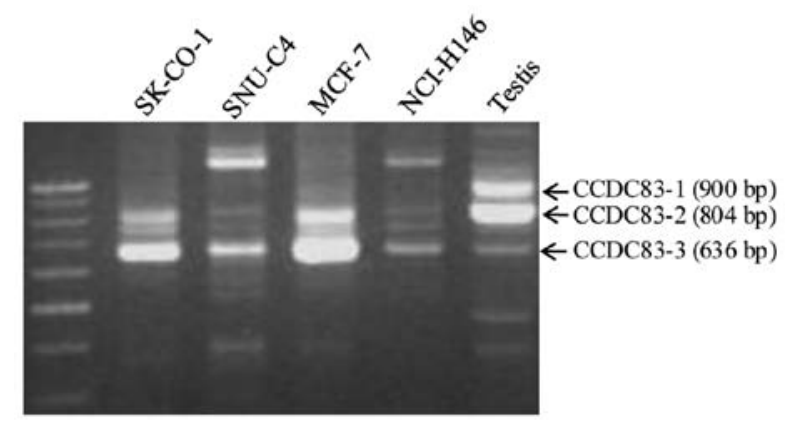

Figure 1. Analysis of CCDC83 gene structures by RT-PCR in testis and cancer cell lines. The three bands were confirmed by DNA sequencing. CCDC83-2 has a deletion in exon 8 and CCDC83-3 has deletions in exon 4 and 8 (data not shown).

dilution) overnight at room temperature. The membranes were washed and incubated with horseradish peroxidaseconjugated sheep anti-human IgG antibody (GE Healthcare) (1:3,000 dilution) for $1 \mathrm{~h}$ at room temperature. After washing with TBST and incubating with chemiluminescence reagent plus (PerkinElmer, Waltham, MA, USA), the membrane was exposed to Kodak medical X-ray film.

\section{Results}

Identification of colon cancer antigens by SEREX. A testis cDNA expression library containing approximately $2 \times 10^{5}$ clones was immunoscreened with serum from a colon cancer patient immunized with dendritic cells. As shown in Table I, 64 clones representing 40 genes were isolated, and the antigens were designated the names KP-CoT-1 through KP-CoT-40.

When the cDNA sequences encoding the 40 colon cancer antigens were compared to those in the Cancer Immunome Database, 17 of the 40 antigens (43\%) had been previously identified by SEREX analysis with any cDNA/serum combination, while the remaining $23(57 \%)$ had not been previously reported (Table I). These included 3 genes with testis-specific expression in the UniGene database (KP-CoT-11, KP-CoT23, and KP-CoT-27). Among the 3 genes with testis-specific profiles, KP-CoT-11, corresponding to sperm acrosomeassociated protein (SPACA7), which was represented by 2 overlapping clones, was isolated. KP-CoT-27 was previously reported as a CT antigen, CAGE (23). KP-CoT-23 was identical to coiled-coil domain containing 83 (CCDC83). KP-CoT-2 (GKAP1), which was represented by 15 clones, was the most frequently isolated gene. Although GKAP1 mRNA expression was observed in some normal tissues, the highest expression was observed in the testis as previously reported (24).

Characterization of the KP-CoT-23 clone. The sequence of the isolated 860-base KP-CoT-23 clone completely matched bases 370 to 1,239 in the 2,345-bp sequence of CCDC83. Comparison with genome databases showed that CCDC83 has 3 splice variants of 2,345 bp (CCDC83-1, NM_173556), 2,035 bp (CCDC83-2, BC040208), and 1,030 bp (CCDC83-3, AY251167), which encode for proteins of 444, 413, and 314 amino acids, respectively. The CCDC83 gene is approximately $52 \mathrm{~kb}$ and contains 11 exons, whereas CCDC83-2 has a deletion in exon 8 and CCDC83-3 has deletions in exons 4 and 8. To 


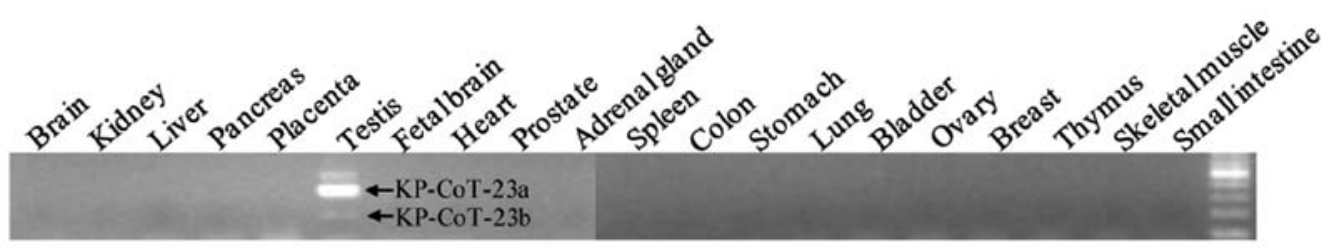

$-\infty-\infty-\infty-\infty,-\infty-\infty,-\infty$

Figure 2. RT-PCR analysis of KP-CoT-23 mRNA in normal tissues. The cDNA templates were normalized using GAPDH as shown in the bottom panel. A band that appears on KP-CoT-23a was confirmed genomic DNA contamination by DNA sequencing.

A
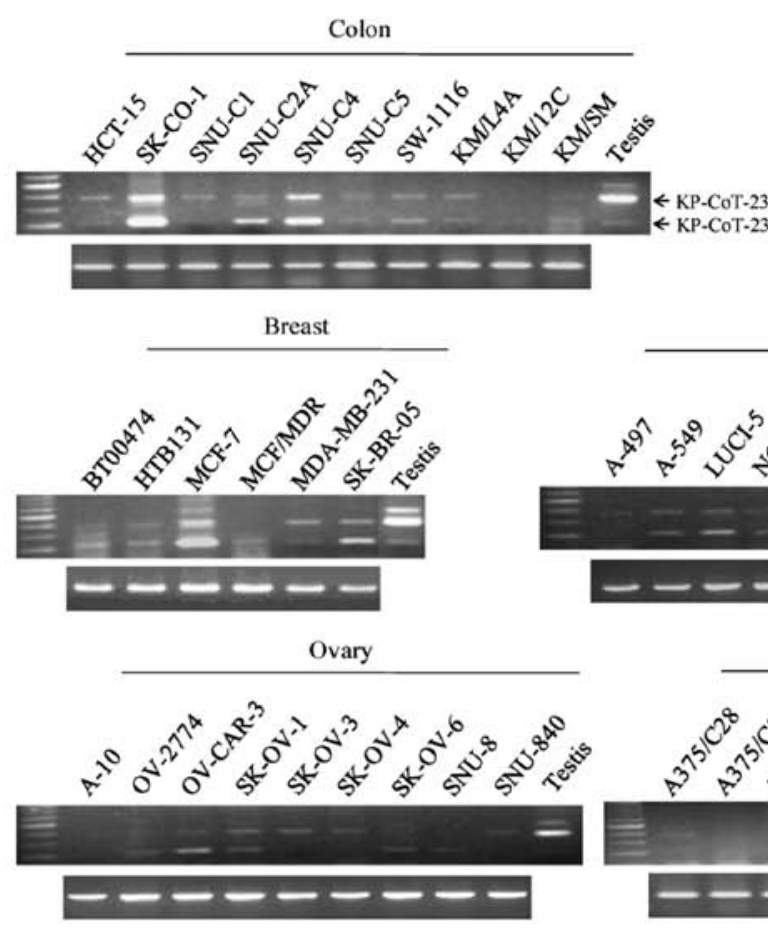

B

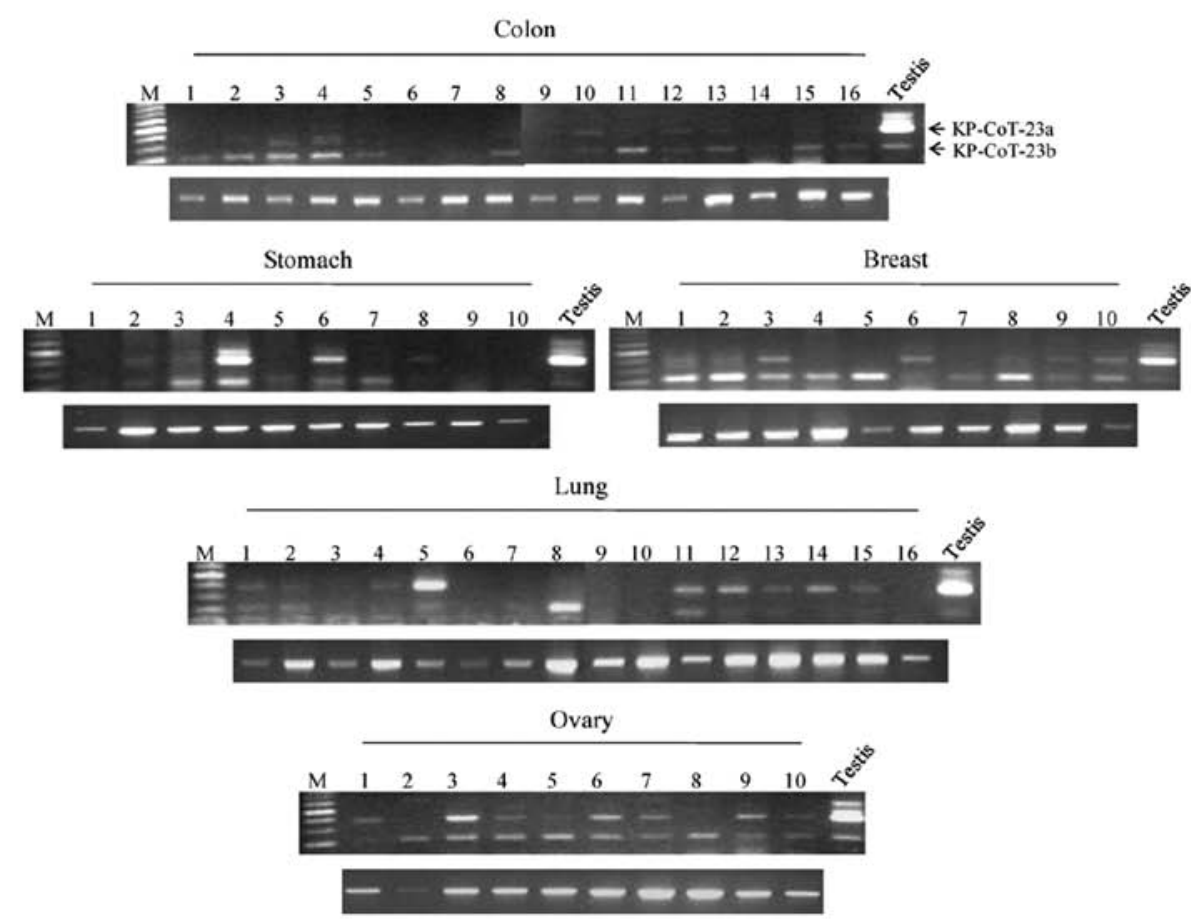

Figure 3. The mRNA expression of KP-CoT-23 in tumors and cancer cell lines. RT-PCR of KP-CoT-23 gene in (A) cancer cells and (B) tumors. The cDNA templates were normalized using GAPDH as shown in the bottom panel. 
Table II. Summary of KP-CoT-23 a and b expression in cancer cell lines.

\begin{tabular}{lccccc}
\hline \multirow{2}{*}{ Tumor type } & \multicolumn{2}{c}{ KP-CoT-23a } & & \multicolumn{2}{c}{ KP-CoT-23b } \\
\cline { 2 - 3 } \cline { 5 - 6 } & Cell lines & Tissues & & Cell lines & Tissues \\
\cline { 2 - 3 } \cline { 5 - 6 } & \multicolumn{2}{c}{ Positive/total } & & \multicolumn{2}{c}{ Positive/total } \\
\hline Breast cancer & $5 / 6$ & $4 / 10$ & & $6 / 6$ & $9 / 10$ \\
Colon cancer & $9 / 10$ & $4 / 16$ & & $10 / 10$ & $12 / 16$ \\
Hepatoma & $1 / 3$ & ND & & $3 / 3$ & ND \\
Lung cancer & $8 / 10$ & $9 / 16$ & & $8 / 10$ & $6 / 16$ \\
Melanoma & $3 / 9$ & ND & & $2 / 9$ & ND \\
Ovary cancer & $6 / 9$ & $9 / 10$ & & $5 / 9$ & $10 / 10$ \\
Stomach cancer & ND & $3 / 10$ & & ND & $4 / 10$ \\
Sarcoma & $3 / 6$ & ND & & $6 / 6$ & ND \\
Leukemia & $2 / 4$ & ND & & $3 / 4$ & ND \\
Prostate cancer & $1 / 3$ & ND & & $3 / 3$ & ND \\
Renal cancer & $2 / 2$ & ND & & $2 / 2$ & ND \\
Glioblastoma & $0 / 2$ & ND & & $2 / 2$ & ND \\
Bladder cancer & $1 / 1$ & ND & & $1 / 1$ & ND \\
Teratoma & $0 / 1$ & ND & & $1 / 1$ & ND \\
\hline
\end{tabular}

ND, not determined.

verify these 3 variants, we performed RT-PCR using specific primer sets that included the deletion regions of testis and several cancer cell lines. As shown in Fig. 1, RT-PCR analysis yielded the expected 3 bands, including a major 800-bp band (CCDC83-2), in testis, whereas only one band (CCDC83-3) or two bands (CCDC83-2 and -3) were detected in the colon cancer cell line SK-CO-1, the breast cancer cell line MCF7, and the small lung cancer cell line NCI-H146. No CCDC83-1 mRNA expression was observed in the cancer cell lines tested. Each band was cloned and sequenced. We confirmed that CCDC83-2 has a deletion in exon 8 and CCDC83-3 has deletions in exons 4 and 8 (data not shown). The KP-CoT-23 clone isolated matched exons $1-5$ of the 11 exons in the CCDC83 gene. In this region, $2 \mathrm{KP}-\mathrm{CoT}-23$ variants were named KP-CoT-23a, which included 5 exons, and KP-CoT-23b, which had 4 exons (deletion of exon 4 in KP-CoT-23a).

KP-CoT-23 mRNA expression in normal tissues, tumors, and cancer cell lines. To investigate the restricted expression of KP-CoT-23 variant mRNAs in normal adult tissues, RT-PCR was performed using gene specific primer pairs to detect KP-CoT-23a and KP-CoT-23b. As shown in Fig. 2, expression of the $2 \mathrm{KP}-\mathrm{CoT}-23$ variant mRNAs was restricted to the testis. KP-CoT-23a was strongly expressed in testis, while KP-CoT-23b was weakly expressed. The KP-CoT-23a and KP-CoT-23b genes were frequently and broadly expressed in a variety of cancer cell lines, including colon cancer cell lines $(9 / 10$ and 10/10, respectively), hepatoma cell lines ( $1 / 3$ and $3 / 3$, respectively), breast cancer cell lines (5/6 and $6 / 6$, respectively), lung cancer cell lines ( $8 / 10$ for both), ovary cancer cell lines (6/9 and 5/9, respectively), melanoma cell lines (3/9 and $2 / 9$, respectively), renal cancer cell lines (1/2), sarcoma cell lines (3/6 and $6 / 6$, respectively), and renal cancer cell lines (2/2 for both) (Fig. 3A, Table II). In addition, the KP-CoT-23a and KP-CoT-23b genes were expressed in various tumors, including colon cancer (4/16 and 12/16, respectively), stomach tumors $(3 / 10$ and $4 / 10$, respectively), breast cancer (4/10 and $9 / 10$, respectively), lung cancer (9/16 and 6/16, respectively), and ovarian cancer (9/10 and 10/10, respectively) (Fig. 3B). These results indicate that KP-CoT-23 is a novel cancer testis antigen that is frequently expressed in several types of cancer, including colon cancer.

Seroreactivity of KP-CoT-23 by western blot analysis. To determine whether immune recognition of the KP-CoT-23 protein is cancer-related, allogeneic sera samples obtained from 21 healthy blood donors and 37 patients with colon cancer were tested for KP-CoT-23 reactivity by western blot analysis. As shown in Fig. 4A and B, 26/37 (70\%) sera from colon cancer patients and 4/21 (19\%) sera from healthy patients were reactive against KP-CoT-23. The correlation between KP-CoT-23 mRNA expression and positive IgG was not evaluated due to a lack of paired samples; therefore, this requires further investigation in a future study. Nonetheless, the results of KP-CoT-23 recognition by sera from colon cancer patients

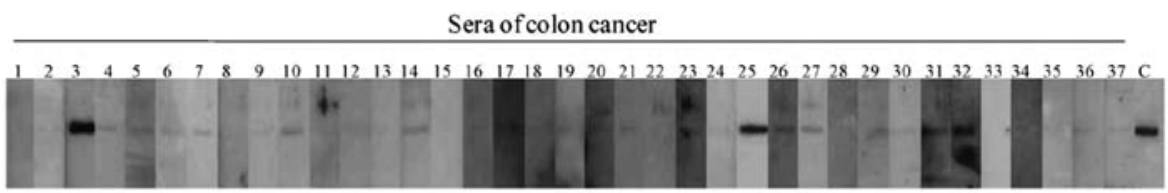

B

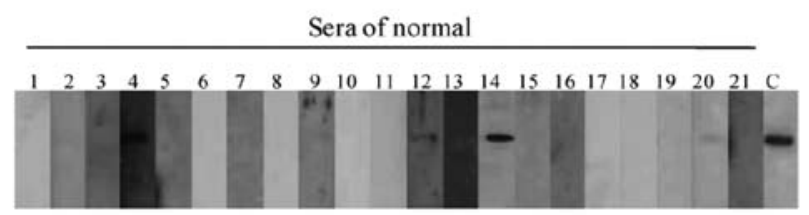

C

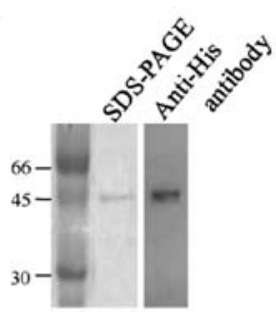

Figure 4. Frequent detection of anti KP-CoT-23 antibodies in sera from colon cancer patients by western blots. Western blotting was performed with the recombinant KP-CoT-23 protein using (A) 37 ovary cancer sera, and (B) 21 normal sera. C indicates control serum, which was used by this SEREX method. (C) The recombinant KP-CoT-23 protein was determined with SDS-PAGE and western blotting using anti-His antibody. 
and healthy individuals indicate that KP-CoT-23 is an immunogenic tumor antigen in colon cancer patients.

\section{Discussion}

In the current study, 40 distinct antigens were isolated from colon cancer by SEREX and were designated the names KP-CoT-1 to KP-CoT-40. Twenty-three of the 40 antigens (57\%) had not been previously identified by SEREX analysis with any cDNA/serum combination. There were 3 genes with testis-specific expression in the UniGene database and in the literature, including KP-CoT-11 (SPACA7), KP-CoT-23 (CCDC83), and KP-CoT-27 (CAGE), a CT antigen previously identified by SEREX.

KP-CoT-11 (SPACA7) is an uncharacterized sperm acrosome-associated protein. Conventional RT-PCR demonstrated strong SPACA7 mRNA expression; however, transcripts encoding SPACA7 were not detected in cancer cell lines and tumor tissues (data not shown).

KP-CoT-27 (CAGE) was previously reported as a CT antigen located on chromosome Xp22. CAGE was highly expressed in several cancer types, including gastric cancer, cervical cancer, and lung cancer (25). Based on an ELISA analysis, anti-CAGE antibodies were detected in sera from 12 of 45 endometrial cancer patients, 2 of 20 melanoma patients, and 4 of 33 colon cancer patients. Although CAGE was isolated from gastric and endometrial cancer, we were the first to find it in colon cancer by SEREX. Detection of anti-CAGE antibody in 7 of $13(53.8 \%)$ patients with microsatellite instability-positive endometrial cancer and in 1 of 3 patients with atypical endometrial hyperplasia (26) suggests that CAGE may be useful for the prognosis or early diagnosis of patients with microsatellite instability-positive endometrial cancers. The expression of CAGE is cell cycle-regulated (25) and confers drug resistance by regulating expression of $\mathrm{p} 53$ through HDAC2 (27).

The KP-CoT-23 gene matched coiled-coil domain containing 83 (CCDC83). The CCDC83 gene consists of 11 exons and has 3 variants, CCDC83-1, CCDC83-2, and CCDC-83-3. RT-PCR analysis revealed that expression of the 3 variants was restricted to the testis in normal adult tissues. In cancer cell lines, no expression of CCDC83-1 mRNA was observed, but expression of CCDC83-2 and CCDC83-3 mRNA was observed in several cancer cell lines (Fig. 1).

The isolated KP-CoT-23 clone matched exons 1-5 of the 11 exons of the CCDC83 gene, and there were 2 KP-CoT-23 variants, named KP-CoT-23a and KP-CoT-23b. The KP-CoT-23a, and $b$ genes were frequently expressed in several tumor types and cancer cell lines, especially in colon cancer tumor $(4 / 16$ and $12 / 16$, respectively) and colon cancer cell lines (9/10 and 10/10, respectively) (Table II). Since CT antigen expression rarely exceeds $40 \%$ in a given cancer type $(2,28)$ and is generally heterogeneous (29), the expression profile of this CT antigen in cancer cells was extraordinary. SEREX-derived CT antigens have been shown to induce $\mathrm{CD}^{+} \mathrm{CTLs}(30,31)$, and a positive correlation was observed between serum positivity for IgG antibody and induction of $\mathrm{CD}^{+} \mathrm{CTL}$ against the cancer testis antigen NY-ESO-1 (31). Western blot analysis of 37 sera samples from colon cancer patients showed that 23 patients had reactivity against the recombinant KP-CoT-23. The signif- icant frequency of IgG antibody responses against KP-CoT-23 suggested that the strong immunogenicity and CD4 and CD8 T-cell responses against the antigen should be investigated.

In summary, a novel CT antigen, KP-CoT-23 was expressed in various types of cancer, including colon cancer. Frequent detection of specific serum IgG antibody in patients with colon cancer indicated the highly immunogenic nature of KP-CoT-23 in colon cancer. These results suggest that KP-CoT-23 may be useful not only for the immunotherapy of colon cancer, but also for the diagnosis of some types of cancer, particularly colon cancer.

\section{Acknowledgements}

The study was supported by a grant from the Basic Research Program of the Korea Science and Engineering Foundation (R01-2004-000-10224-02006) and a grant from the Cancer Research Institute, USA.

\section{References}

1. Scanlan MJ, Gure AO, Jungbluth AA, Old LJ and Chen YT: Cancer/testis antigens: an expanding family of targets for cancer immunotherapy. Immunol Rev 188: 22-32, 2002.

2. Caballero OL and Chen YT: Cancer/testis (CT) antigens: potential targets for immunotherapy. Cancer Sci 100: 2014-2021, 2009.

3. Simpson AJ, Caballero OL, Jungbluth A, Chen YT and Old J: Cancer/testis antigens, gametogenesis and cancer. Nat Rev Cancer 5: 615-625, 2005.

4. Kim YD, Park HR, Song MH, Shin DH, Lee CH, Lee MK and Lee SY: Pattern of cancer/testis antigen expression in lung cancer patients. Int J Mol Med 29: 656-662, 2012.

5. Gaugler B, Van den Eynde B, van der Bruggen P, Romero P, Gaforio JJ, De Plaen E, Lethe B, Brasseur F and Boon T: Human gene MAGE-3 codes for an antigen recognized on a melanoma by autologous cytolytic T lymphocytes. J Exp Med 179: 921-930, 1994.

6. van der Bruggen $\mathrm{P}$, Traversari $\mathrm{C}$, Chomez $\mathrm{P}$, Lurquin $\mathrm{C}$, De Plaen E, Van den Eynde B, Knuth A and Boon T: A gene encoding an antigen recognized by cytolytic $\mathrm{T}$ lymphocytes on a human melanoma. Science 254: 1643-1647, 1991.

7. Pascolo S, Schirle M, Guckel B, Dumrese T, Stumm S, Kayser S, Moris A, Wallwiener D, Rammensee HG and Stevanovic S: A MAGE-A1 HLA-A A*0201 epitope identified by mass spectrometry. Cancer Res 61: 4072-4077, 2001.

8. Sahin U, Tureci O, Schmitt H, Cochlovius B, Johannes T, Schmits R, Stenner F, Luo G, Schobert I and Pfreundschuh M: Human neoplasms elicit multiple specific immune responses in the autologous host. Proc Natl Acad Sci USA 92: 11810-11813, 1995.

9. de Wit NJ, Weidle UH, Ruiter DJ and van Muijen GN: Expression profiling of MMA-1a and splice variant MMA-1b: new cancer/ testis antigens identified in human melanoma. Int J Cancer 98: 547-553, 2002.

10. Chen YT, Scanlan MJ, Venditti CA, Chua R, Theiler G, Stevenson BJ, Iseli C, Gure AO, Vasicek T, Strausberg RL, et al: Identification of cancer/testis-antigen genes by massively parallel signature sequencing. Proc Natl Acad Sci USA 102: 7940-7945, 2005.

11. Scanlan MJ, Altorki NK, Gure AO, Williamson B, Jungbluth A, Chen YT and Old LJ: Expression of cancer-testis antigens in lung cancer: definition of bromodomain testis-specific gene (BRDT) as a new CT gene, CT9. Cancer Lett 150: 155-164, 2000.

12. Chen YT, Venditti CA, Theiler G, Stevenson BJ, Iseli C, Gure AO, Jongeneel CV, Old LJ and Simpson AJ: Identification of CT46/ HORMAD1, an immunogenic cancer/testis antigen encoding a putative meiosis-related protein. Cancer Immun 5: 9, 2005.

13. Brinkmann U, Vasmatzis G, Lee B and Pastan I: Novel genes in the PAGE and GAGE family of tumor antigens found by homology walking in the dbEST database. Cancer Res 59: 1445-1448, 1999. 
14. Sato S, Noguchi Y, Ohara N, Uenaka A, Shimono M, Nakagawa K, Koizumi F, Ishida T, Yoshino T, Shiratori Y and Nakayama E: Identification of XAGE-1 isoforms: predominant expression of XAGE-1b in testis and tumors. Cancer Immun 7: 5, 2007.

15. Lee SY, Obata Y, Yoshida M, Stockert E, Williamson B, Jungbluth AA, Chen YT, Old LJ and Scanlan MJ: Immunomic analysis of human sarcoma. Proc Natl Acad Sci USA 100: 2651-2656, 2003.

16. Chen YT, Gure AO, Tsang S, Stockert E, Jager E, Knuth A and Old LJ: Identification of multiple cancer/testis antigens by allogeneic antibody screening of a melanoma cell line library. Proc Natl Acad Sci USA 95: 6919-6923, 1998.

17. Chen YT, Scanlan MJ, Sahin U, Tureci O, Gure AO, Tsang S, Williamson B, Stockert E, Pfreundschuh M and Old LJ: A testicular antigen aberrantly expressed in human cancers detected by autologous antibody screening. Proc Natl Acad Sci USA 94: 1914-1918, 1997.

18. Tureci O, Sahin U, Schobert I, Koslowski M, Scmitt H, Schild HJ, Stenner F, Seitz G, Rammensee HG and Pfreundschuh M The SSX-2 gene, which is involved in the $\mathrm{t}(\mathrm{X} ; 18)$ translocation of synovial sarcomas, codes for the human tumor antigen HOM-MEL-40. Cancer Res 56: 4766-4772, 1996.

19. Tureci O, Sahin U, Zwick C, Koslowski M, Seitz G and Pfreundschuh M: Identification of a meiosis-specific protein as a member of the class of cancer/testis antigens. Proc Natl Acad Sci USA 95: 5211-5216, 1998 .

20. Lee SY, Williamson B, Caballero OL, Chen YT, Scanlan MJ, Ritter G, Jongeneel CV, Simpson AJ and Old LJ: Identification of the gonad-specific anion transporter SLCO6A1 as a cancer/testis (CT) antigen expressed in human lung cancer. Cancer Immun 4: 13, 2004.

21. Park S, Lim Y, Lee D, Cho B, Bang YJ, Sung S, Kim HY, Kim DK, Lee YS, Song Y and Jeoung DI: Identification and characterization of a novel cancer/testis antigen gene CAGE-1. Biochim Biophys Acta 1625: 173-182, 2003.

22. Song MH, Ha JC, Lee SM, Park YM and Lee SY: Identification of BCP-20 (FBXO39) as a cancer/testis antigen from colon cancer patients by SEREX. Biochem Biophys Res Commun 408: 195-201, 2011

23. Cho B, Lim Y, Lee DY, Park SY, Lee H, Kim WH, Yang H, Bang Y $J$ and Jeoung DI: Identification and characterization of a novel cancer/testis antigen gene CAGE. Biochem Biophys Res Commun 292: 715-726, 2002.
24. Domae S, Nakamura Y, Uenaka A, Wada H, Nakata M, Oka M, Kishimoto K, Tsukamoto G, Yoshihama Y, Matsuoka J, et al: Identification of CCDC62-2 as a novel cancer/testis antigen and its immunogenicity. Int J Cancer 124: 2347-2352, 2009.

25. Kim Y and Jeoung D: Role of CAGE, a novel cancer/testis antigen, in various cellular processes, including tumorigenesis, cytolytic T lymphocyte induction, and cell motility. J Microbiol Biotechnol 18: 600-610, 2008.

26. Iwata T, Fujita T, Hirao N, Matsuzaki Y, Okada T, Mochimaru H, Susumu N, Matsumoto E, Sugano K, Yamashita N, et al: Frequent immune responses to a cancer/testis antigen, CAGE, in patients with microsatellite instability-positive endometrial cancer. Clin Cancer Res 11: 3949-3957, 2005.

27. Kim Y, Park H, Park D, Lee YS, Choe J, Hahn JH, Lee H, Kim YM and Jeoung D: Cancer/testis antigen CAGE exerts negative regulation on p53 expression through HDAC2 and confers resistance to anti-cancer drugs. J Biol Chem 285: 25957-25968, 2010.

28. Jungbluth AA, Chen YT, Stockert E, Busam KJ, Kolb D, Iversen K, Coplan K, Williamson B, Altorki N and Old LJ: Immunohistochemical analysis of NY-ESO-1 antigen expression in normal and malignant human tissues. Int J Cancer 92: 856-860, 2001.

29. Slingluff CL Jr, Petroni GR, Chianese-Bullock KA, Smolkin ME, Hibbitts S, Murphy C, Johansen N, Grosh WW, Yamshchikov GV, Neese PY, et al: Immunologic and clinical outcomes of a randomized phase II trial of two multipeptide vaccines for melanoma in the adjuvant setting. Clin Cancer Res 13: 63866395, 2007

30. Coulie PG, Karanikas V, Colau D, Lurquin C, Landry C, Marchand M, Dorval T, Brichard V and Boon T: A monoclonal cytolytic T-lymphocyte response observed in a melanoma patient vaccinated with a tumor-specific antigenic peptide encoded by gene MAGE-3. Proc Natl Acad Sci USA 98: 10290-10295, 2001.

31. Jager E, Nagata Y, Gnjatic S, Wada H, Stockert E, Karbach J, Dunbar PR, Lee SY, Jungbluth A, Jager D, et al: Monitoring CD8 T cell responses to NY-ESO-1: correlation of humoral and cellular immune responses. Proc Natl Acad Sci USA 97: 4760-4765, 2000. 